\title{
High level of unawareness of diabetes in the Kuwaiti population
}

Abdullah Alkandari, Joseph Longenecker, Ahmad Alkhatib, Rihab Al-Wotayan,

Noël C Barengo, Qais Al Duwairi and Jaakko Tuomilehto

Dasman Diabetes Institute, Kuwait

\section{INTRODUCTION}

- The Middle East and North Africa (MENA) have been heavily hit by the global diabetes epidemic.

- However, the percentage of adults with diabetes unaware of their illness is unknown.

- A national survey of adults was conducted in Kuwait in 2014 to determine the prevalence of non-communicable diseases and their risk factors. Here, we outline the prevalence of unawareness of diabetes in Kuwaiti adults.

\section{METHODS}

- 3915 Kuwaiti men and women aged 18-69 were surveyed using the WHO Instrument for Chronic Disease Risk Factor Surveillance

- Three consecutive steps:

I. Demographics and medical history

II. Physical measurements

III. Blood biochemistry, including fasting plasma glucose and HbA1c

- Diabetes unawareness was defined as having a FPG $\geq 7 \mathrm{mmol} / \mathrm{L}$ or an $\mathrm{HbA} 1 \mathrm{c} \geq 6.5 \%$ among participants reporting that they did not have a previous diagnosis of diabetes.

\begin{tabular}{|c|c|c|c|}
\hline \multicolumn{4}{|c|}{ RESULTS } \\
\hline \multicolumn{4}{|c|}{ Table 1: Participant characteristics } \\
\hline & Total & Male & Female \\
\hline $\mathrm{n}$ & 3915 (100) & $1456(37.2)$ & $2459(62.8)$ \\
\hline Age (Years) & $37.2(12.3)$ & $36.9(12.6)$ & $37.3(12)$ \\
\hline $\mathrm{BMI}\left(\mathrm{kg} / \mathrm{m}^{2}\right)$ & $29.6(6.15)$ & $29.2(5.62)$ & $29.9(6.44)$ \\
\hline Waist Circumference (cm) & $90.7(15.2)$ & $94.2(15.2)$ & $88.6(14.8)$ \\
\hline Hip Circumference (cm) & $106.6(14.6)$ & $105.8(14.3)$ & $107.1(14.8)$ \\
\hline Fasting Plasma Glucose (mmol/l) & $5.74(2.43)$ & $5.9(2.47)$ & $5.65(2.39)$ \\
\hline HbA1c (\%) & $5.81(1.36)$ & $5.83(1.34)$ & $5.80(1.37)$ \\
\hline
\end{tabular}

Figure 1: Diabetes and pre-diabetes prevalence

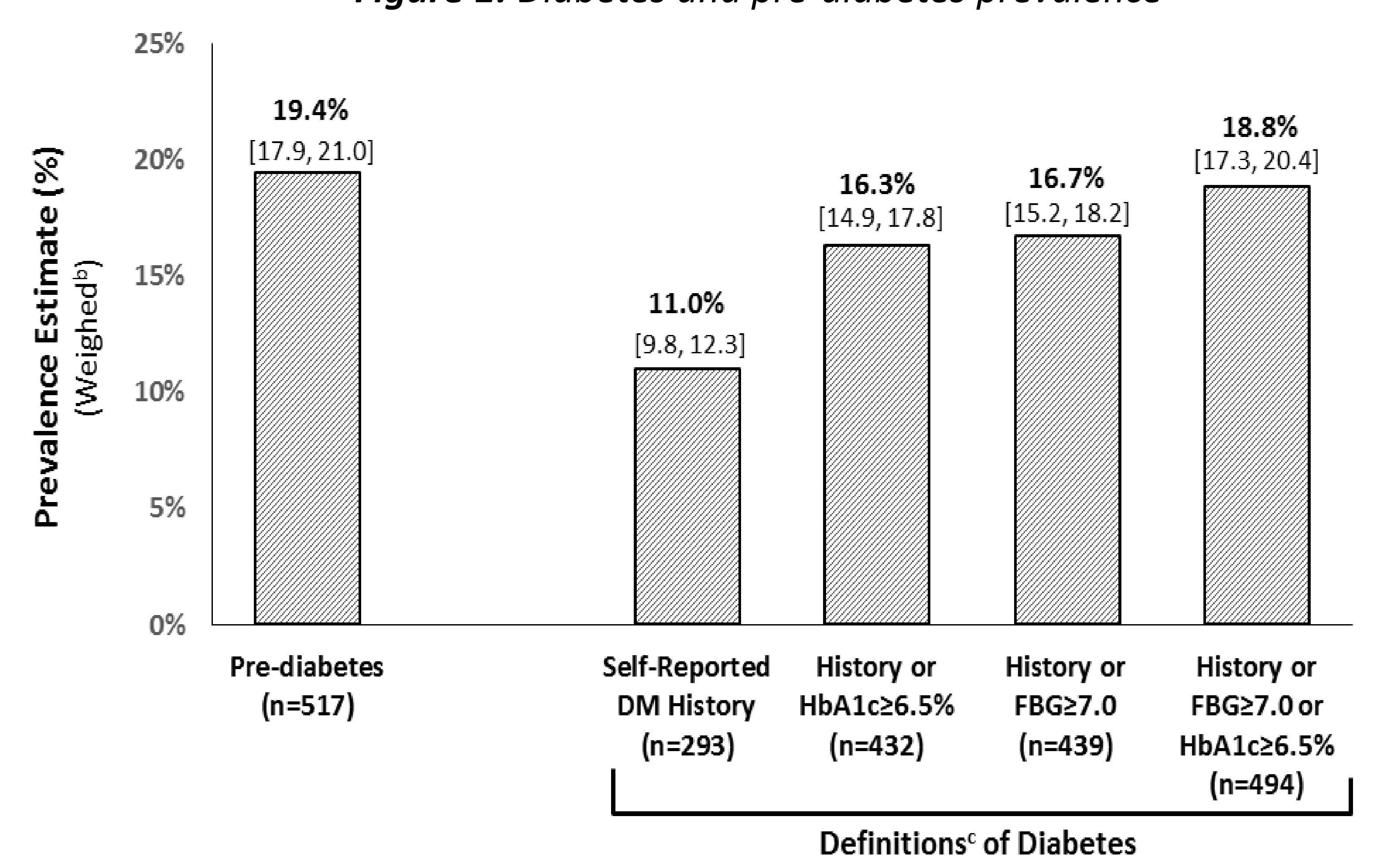

\section{RESULTS}

Table 2: Percent diabetes unawareness according to age, sex, and BMI group

\begin{tabular}{|c|c|c|c|c|c|c|c|}
\hline \multirow[t]{2}{*}{ Characteristic } & \multirow{2}{*}{$\begin{array}{c}\begin{array}{c}\text { Sub- } \\
\text { group }\end{array} \\
\mathrm{N} \\
\end{array}$} & \multicolumn{3}{|c|}{$\begin{array}{c}\text { Percent Unawareness } \\
\text { of Diabetes }\end{array}$} & \multicolumn{3}{|c|}{$\begin{array}{l}\text { Adjusted Odds Ratio } \\
\text { Of Unawareness }\end{array}$} \\
\hline & & $n$ & $(\%)$ & $\mathrm{P}$ & OR & {$[95 \% \mathrm{Cl}]$} & $\mathrm{P}$ \\
\hline All & 494 & 201 & (41.5) & & N/A & & \\
\hline Age group & & & & $<0.001$ & & & \\
\hline $18-29$ & 50 & 32 & (63.5) & & 1.0 & [Reference] & \\
\hline $30-44$ & 135 & 78 & (59.1) & & 0.9 & {$[0.4,1.8]$} & 0.70 \\
\hline $45-59$ & 219 & 70 & (32.4) & & 0.3 & {$[0.2,0.6]$} & $<0.001$ \\
\hline $60-69$ & 90 & 21 & (23.0) & & 0.2 & {$[0.15,0.6]$} & $<0.001$ \\
\hline Gender & & & & 0.06 & & & \\
\hline Male & 210 & 94 & $(45.3)$ & & 1.0 & [Reference] & \\
\hline Female & 284 & 107 & (37.4) & & 0.8 & {$[0.6,1.3]$} & 0.41 \\
\hline BMI Group & & & & 0.08 & & & \\
\hline$<25 \mathrm{~kg} / \mathrm{m}^{2}$ & 46 & 21 & $(47.2)$ & & 1.0 & [Reference] & \\
\hline $25-29 \mathrm{~kg} / \mathrm{m}^{2}$ & 129 & 63 & (48.9) & & 1.2 & {$[0.6,2.5]$} & 0.62 \\
\hline $30-34 \mathrm{~kg} / \mathrm{m}^{2}$ & 166 & 55 & $(34.0)$ & & 0.8 & {$[0.4,1.6]$} & 0.49 \\
\hline$\geq 35 \mathrm{~kg} / \mathrm{m}^{2}$ & 153 & 62 & (41.1) & & 1.0 & {$[0.5,2.0]$} & 0.98 \\
\hline
\end{tabular}

Figure 2: Diabetes unawareness by sex and age

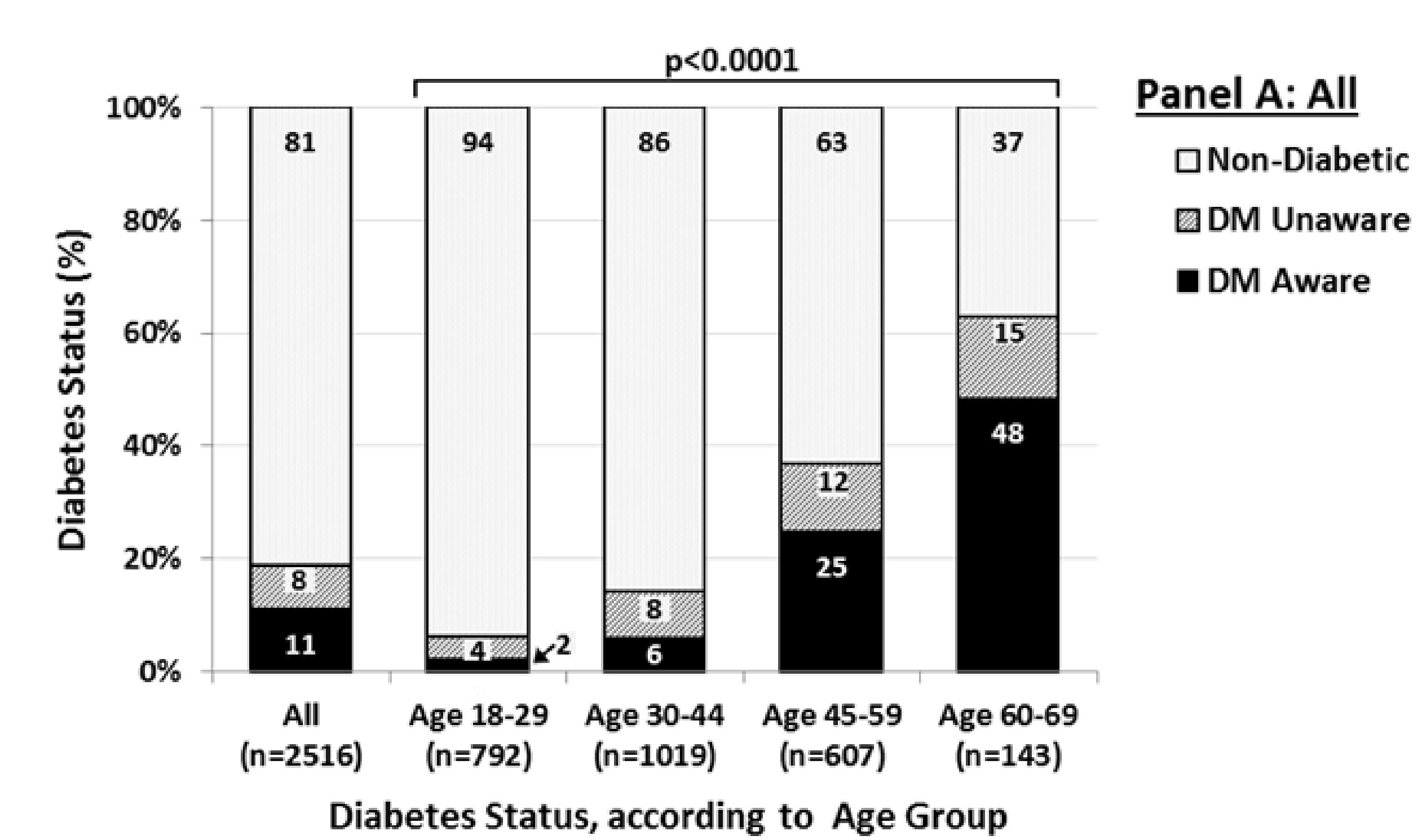

Diabetes Status, according to Age Group
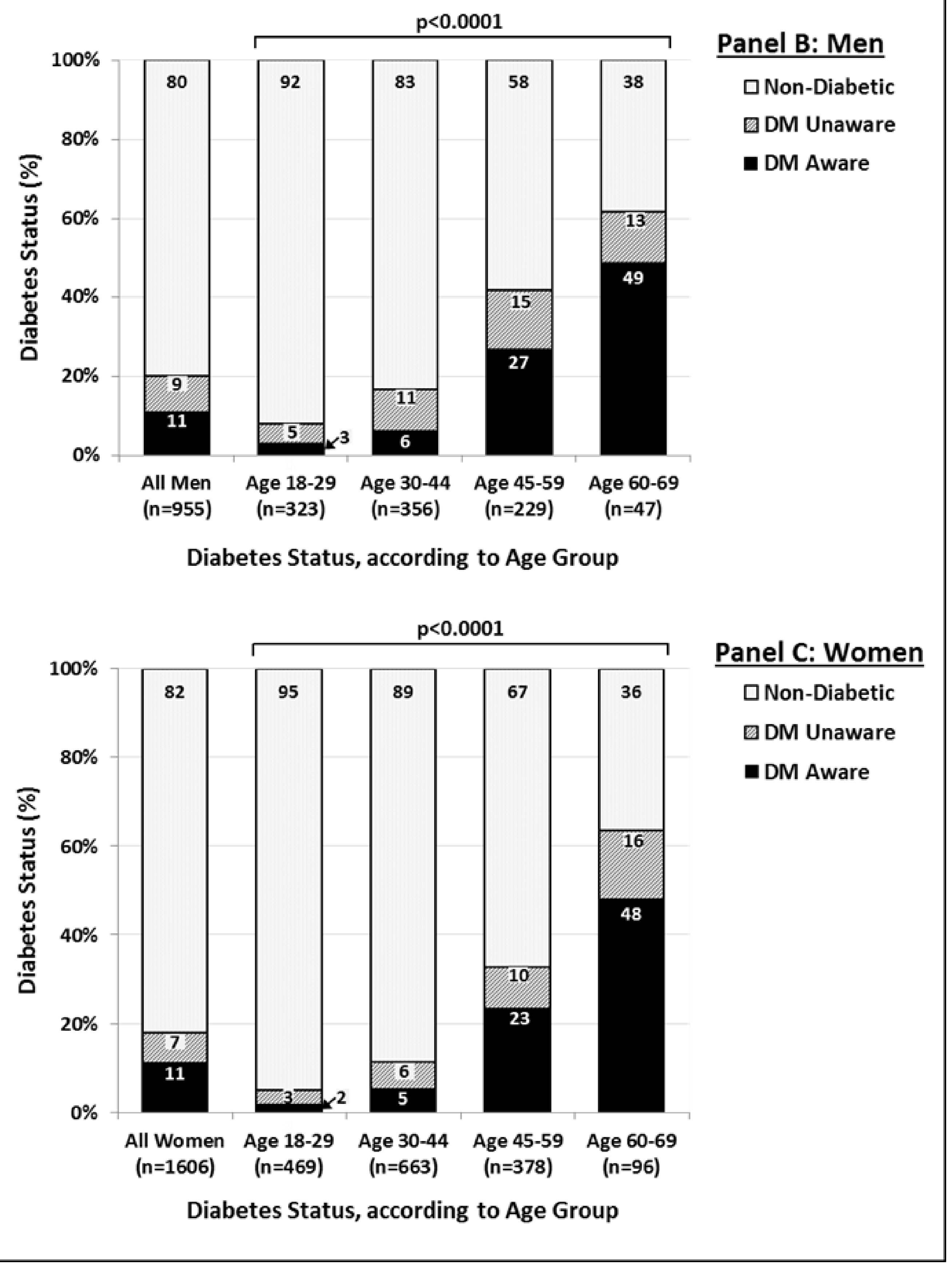

CONCLUSION

Over one third of Kuwaiti adults with diabetes are unaware of their disease 